\title{
Estudio sobre el caso de la producción creciente del tomate en los desiertos mediante el sistema agrario con poco insumo: desafíos en la zona costera del Perú
}

\section{Study on the growing production case of the tomato in the deserts by the agrarian system with not much material: challenges in the Peru's coastal zone}

\author{
Koichi Numata, ${ }^{*}$ Keishiro Itagaki ${ }^{* *}$ \\ (Con el apoyo y colaboración de Vicente Rázuri, Andrés Casas y Francisco Delgado de la Flor.)
}

http://dx.doi.org/10.21503/CienciayDesarrollo.2011.v14.02

\section{RESUMEN}

En la actualidad, el tomate es la hortaliza de mayor consumo en el mundo, ya que su producción total al año alcanza los 130 millones de toneladas. La demanda ha ido aumentando significativamente por el creciente reconocimiento de su valor nutritivo, representado por el licopeno, entre otros. De hecho, la industria del tomate está generalmente concentrada en el área llamada la faja del tomate, situada en la zona semitropical, entre los 30 y $40^{\circ}$ de latitud norte y los 30 y 40 grados de latitud sur.

Sin embargo, nos parece difícil incrementar la producción del tomate aún más en esta zona, debido a la aguda competencia en torno a la distribución de recursos entre el tomate y otros productos agrícolas. En cambio, en la zona baja y tropical del Perú, situada entre los 0 y 30 grados, se ha reportado que la producción del pimiento páprika (que pertenece a la misma categoría botánica del tomate) ha prosperado exitosamente. Por otra parte, en la industria del tomate en el Perú, se han presentado diferentes fracasos consecutivos hasta el año 2000. No obstante, en el Proyecto ASKA (Proyecto Experimental de Tomate, realizado en la zona de Chavimochic, en Trujillo, con la participación de la Empresa Nacional KAGOMEJapón), llevado a cabo con la estrecha colaboración entre la industria del tomate y la Universidad Nacional Agraria La Molina, se pudo comprobar que el cultivo del tomate industrial era factible en la costa peruana. Por otra parte, ICATOM, que no participó en dicho proyecto, logró éxitos en el cultivo del tomate industrial en el sur del Perú.

En el presente trabajo, comparando el caso exitoso del pimiento páprika y los casos de fracaso en el cultivo del tomate industrial en el Perú, se espera señalar concisamente los factores que sirvan para que la industria del tomate en el Perú logre alcanzar éxitos, al mismo tiempo de obtener conocimientos e informaciones precisas relacionados con la producción del tomate a escala comercial en los desiertos peruanos.

Palabras clave: tomate, riego por goteo, polinización, alcalinización, licopeno.

\section{ABSTRACT}

Now, the tomato is the vegetable of more consumption in the world, since its total production a year reaches the 130 million tons. The demand has gone increasing significantly for the growing recognition of its nutritious value, represented by the licopeno, among other. In fact, the industry of the tomato is

* Máster en Agricultura por Tokyo University of Agriculture. (koichi.numata@scfoods.co.jp)

** Faculty of Food and International Studies, Tokyo University of Agriculture. 
generally concentrated in the called area the strip of the tomato, located in the area semitropical, between the 30 and 40 grades of north latitude and the 30 and 40 grades of south latitude.

However, we find difficult to increase the production of the even more tomato in this area, due to the sharp competition around the distribution of resources between the tomato and other agricultural products. On the other hand, in the low and tropical area of Peru, located in the grades 0-30, it has been reported that the production of the pepper paprika (that it belongs to the botanical same category of the tomato) has prospered successfully. On the other hand, in the industry of the tomato in Peru, they have shown up serial different failures until the year 2000. Nevertheless, in the Project ASKA (Experimental Project of Tomato, carried out in the area of Chavimochic, in Trujillo, with the participation of the National Company KAGOME-Japan), carried out with the narrow collaboration between the industry of the tomato and the National Agrarian University Molina, it could be proven that the cultivation of the industrial tomato was feasible in the Peruvian coast. On the other hand, ICATOM that did not participate in this project, achieved successes in the cultivation of the industrial tomato in the south of Peru.

Presently work, comparing the successful case of the pepper paprika and the cases of failure in the cultivation of the industrial tomato in Peru, it is hoped to point out the factors that serve concisely so that the industry of the tomato in Peru is able to succeed, at the same time of obtaining knowledge and precise informations related with the production from the tomato to commercial scale in the Peruvian deserts.

Key words: tomato, watering for drip, pollination, alkalinization, licopeno.

\section{INTRODUCCIÓN}

El tomate es la verdura de mayor consumo en el mundo, con una producción mundial al año que llega a los 130 millones de toneladas. (1) En años recientes, la demanda de tomate está creciendo aceleradamente no solamente en los países desarrollados sino también en los países emergentes y en vías de desarrollo,(2) porque los valores nutritivos del tomate (minerales, licopeno, entre otros) están llamando la atención del mundo. En el pasado se consideraba que para el cultivo de tomate a la intemperie, el clima subtropical (es decir, el clima mediterráneo) en la faja del tomate (30-40 grados de latitud norte y sur) era el adecuado. Consecuentemente, el cultivo de tomate a la intemperie y la industria manufacturera de tomate en escala comercial se concentran en esta faja climática, favorecida por tener la temperatura más adecuada para la polinización ${ }^{1}$ (20-25 centígrados) y la temperatura acumulada más favorable $^{2}$ (1100-1250 grados desde el inicio de florecimiento hasta la cosecha).(3) Sin embargo, en dicha zona hay muchos cultivos que compiten con tomate, de modo que año tras año escasean más nuevos terrenos adecuados para el cultivo a la intemperie de tomate en gran escala. Por lo tanto, se considera difícil el mayor cultivo de tomate a la intemperie en esta zona desde el punto de vista físico y económico. ${ }^{3}$ Por otra parte, el Perú, país situado en la zona que va de los 0 a 30 grados de latitud sur, ostenta la condición de ser el país de mayor producción de pimentón, que pertenece a la misma familia de la berenjena, y de tomate,

1 Resultado de la temperatura media del día multiplicada por el número de días.

2 De acuerdo con las cifras de la FAO, la producción mundial de tomate al año fue de 130 millones para el 2005, y los primeros 6 países productores de tomate en el mundo son los siguientes: China (19,5\%), Estados Unidos de América (9,5\%), Turquía (6,9\%), India (5,7\%), Egipto (4,8\%) e Italia (4,6\%). La producción sumada de estos 6 países es equivalente al $51 \%$ de toda la producción mundial, y geográficamente estos países están concentrados entre los 30 y 40 grados de latitud norte y sur.

3 Según los datos de FAOSTAT (http://fao.org/landandwater/agil/agromaps/interactive/page.jspx), el cultivo de maíz, que compite con el cultivo del tomate, está creciendo aceleradamente en la misma zona subtropical por la demanda creciente de bioetanol y biodiésel. (Fecha de acceso: 17 de enero de 2011.) 
por el cultivo exitoso a la intemperie en escala comercial. No obstante, a pesar de que el tomate es oriundo de este país, muchas pruebas para su cultivo a la intemperie en escala comercial hechas en el Perú hasta el año 2000 resultaron en fracasos. Sin embargo, la experiencia exitosa lograda por el Proyecto ASKA (sobre este punto se dará mayor explicación más adelante) a partir del año 2003 fue sucedida por la de la empresa ICATOM, la que sufrió una plaga durante su intento de cultivar tomate a la intemperie en Ica, en la parte sur del país; pero al fin esta empresa ha conseguido el éxito con sus negocios de tomate.

Con todo, consideramos que mediante la comparación de los factores causantes del fracaso en el caso del cultivo de tomate a la intemperie en el Perú con los factores favorables para el éxito en el caso del cultivo de pimentón, así como por la identificación de las condiciones adecuadas para el cultivo exitoso de tomate a la intemperie en escala comercial con base en la experiencia exitosa, se está contribuyendo a la ampliación de posibilidades en cuanto al cultivo de tomate en otras zonas bajas con clima tropical, situadas a la misma latitud del Perú.

El objetivo principal de este trabajo es la identificación de las potencialidades y las condiciones que garantizan el éxito en el cultivo de tomate a la intemperie en escala comercial, mediante la comparación de factores de éxito en caso del cultivo del pimentón a la intemperie en escala comercial con los factores causantes de fracaso en el cultivo de tomate a la intemperie en escala comercial, pese a que el tomate pertenece a la misma familia de la berenjena y del pimentón. Asimismo, se tomarán en cuenta los análisis de los antecedentes y factores de algunos casos exitosos del cultivo de tomate en escala comercial.

\section{MATERIALES Y MÉTODOS}

En este texto, son usados los datos de experimentos llevados a cabo en el cultivo de tomate a la intemperie en escala comercial en el marco de un proyecto denominado ASKA, desarrollado durante los años 2000 y 2002, en el cual nosotros, los autores de este trabajo, también participamos. Los datos de los experimentos relativos al cultivo de tomate a la intemperie en escala comercial hecho por la empresa privada ICATOM, fueron conseguidos mediante una serie de gestiones realizadas ante dicha empresa durante los años 1997 y 2002. Asimismo, no debemos dejar de mencionar que algunos documentos de referencia en torno a la industria peruana del tomate fueron elaborados conjuntamente entre el sector industrial y académico.(4) Hasta la fecha no se ha llevado a cabo ningún estudio de esta naturaleza, y este trabajo es el resultado de un conjunto de experiencias nuestras in situ en diferentes trabajos e investigaciones.

\section{Factores de éxito en el cultivo del pimiento} páprika en el Perú

El pimiento no era cultivado en escala comercial en el Perú hasta 1993; sin embargo, desde 1994 comenzó su cultivo a nivel comercial en los desiertos de las costa, y el 2001 la superficie ocupada por este cultivo alcanzó aproximadamente unas 4000 ha.(5) No obstante, la cantidad de cosecha por hectárea era muy fluctuante, variando entre 2 y 6 toneladas al año al estado seco, y consecuentemente no era muy competitivo para la exportación. Hacia el año 2000, la implementación de campos con riego por goteo, ${ }^{4}$ que había sido uno de los factores para el éxito en el cultivo del espárrago, fue introducida en el cultivo de este tipo de

4 Con el riego por goteo se puede regar constantemente todos los días con una cantidad de agua comparativamente menor a la empleada en el riego tradicional por surco. Además, se puede agregar el fertilizante diluido. Por lo general, la cosecha es mayor que con el uso de riego por surco, que consume abundante agua. 
pimiento a campo abierto en gran escala.(6) Esta tecnología contribuyó al mejoramiento de la competitividad del páprika peruano, convirtiéndolo en foco de atención mundial, y como consecuencia de ello, la superficie total dedicada a este tipo de pimiento llegó en el 2005 a 9000 ha.(5)

Los factores que llevaron al éxito el cultivo del pimiento páprika y los retos que tiene que enfrentar se pueden resumir en los siguientes puntos:

\section{Aspectos exitosos}

- Factores relacionados con el medio ambiente (clima adecuado).

- Disponibilidad de agua (ríos, agua subterránea).

- Uso de riego tecnificado (riego por goteo).

- Cultivo intensivo (una campaña al año en el mismo terreno).

- Uso de materia orgánica (estiércol de aves).

Aspectos comerciales involucrados en los aspectos exitosos

- Pérdida de competitividad internacional de España, país exportador de páprika, debido al significativo incremento del costo agrícola.

- Brusco aumento de la competitividad internacional del Perú en el área agrícola, gracias al fomento de cultivos a gran escala comercial, como resultado de la promoción de la agricultura por parte del gobierno peruano.

\section{Retos a futuro}

- Implementación de métodos integrados en el control de plagas y enfermedades.

- Disminución de los costos de producción, que en la actualidad se estiman están cerca de los US \$ 7000,00 por ha debido al uso de muchos pesticidas, que equivalen a unos US $\$ 2000,00$ por ha.

Factores involucrados en el fracaso del cultivo del tomate a campo abierto en el Perú

1. Características del clima en los desiertos de la zona costera peruana

En invierno (junio-setiembre), desde el Océano Pacífico sopla viento frío y húmedo acompañado de neblina, y a veces caen lluvias muy finas denominadas "garúas". Durante estos meses, la temperatura media fluctúa entre $15 \mathrm{y}$ $18^{\circ} \mathrm{C}$, y la humedad media varía entre 70 y $80 \%$.

En cambio, en verano (enero-marzo) atraviesa por la zona costera un viento relativamente cálido que sopla desde el Océano Pacífico. La temperatura se mueve entre los 18 y $23^{\circ} \mathrm{C}$, y la humedad media varía de 70 a $80 \%$. Durante todo el año, las lluvias son muy escasas en los desiertos de la zona costera del Perú, y el clima es principalmente moderado y estable.

\section{Factores de fracaso en el cultivo del tomate (periodo 1980 -2000)}

Durante los años 1980 y 2000 , en siete diferentes lugares en los desiertos de la zona costera peruana se cultivo el tomate con fines industriales. Sin embargo, todas estas inversiones en tomate industrial resultaron en fracasos, por lo que la industria tomatera del Perú se vio obligada enfrentar una situación crítica que puso en riesgo su sobrevivencia.

Analizaremos dos casos representativos de este fracaso:

a. En la década de los 80, en la zona costera del norte, la empresa TOMANOR manejaba más de 1000 ha de tierra agrícola y se dedicaba al cultivo del tomate industrial utilizando el 
riego por aspersión, que a su vez se valía de un pivote al estilo californiano, con abundante uso de pesticidas y fertilizantes químicos. Sin embargo, no fue posible cultivar el tomate de forma estable, lo que trajo como resultado el retiro de la empresa de este negocio. En este caso, consideramos que la causa del fracaso fue un mal manejo empresarial, además de problemas técnicos como la acumulación de sales y posterior alcalinización.(5)

b. En la década de los noventa, la empresa Agroguayabito venía cultivando tomate a lo largo de años sucesivos en la zona costera del centro del Perú. Como resultado, el manejo fitosanitario, en especial de la mosca blanca, se hacía incontrolable, obligando el alto uso de plaguicidas, lo que a su vez trajo desbalances en la población de insectos, con el agregado de que el alto uso de pesticidas afectaba la calidad del suelo y agua. Esto obligó a la empresa a dejar de cultivar el tomate, ya que los rendimientos bajaban año tras año. Estos malos resultados agronómicos produjeron una crisis financiera en la empresa que finalmente la llevó a la quiebra. En este caso, el aspecto fitosanitario fue considerado la causa del fracaso en el intento de cultivar el tomate en forma continua en un mismo lugar o zona.(7)

\section{Comparaciones con el caso delpimientopáprika}

El cultivo del pimiento páprika sembrado a escala comercial en la zona desértica costera peruana fue exitoso porque se supo aplicar una tecnología de riego, en este caso el riego por goteo, evitando problemas con la alta salinidad tanto del agua como del suelo. TOMANOR fracasó en su intento de cultivar tomate industrial a gran escala empleando el sistema de riego por aspersión, tipo PIVOT Central, por los problemas de alcalinización y también de gestión empresarial. Esta experiencia negativa sin lugar a dudas resalta la importancia de usar el tipo de riego adecuado para lograr buenos rendimientos en las condiciones de la costa peruana.
$\mathrm{Si}$ bien es cierto Agroguayabito empleó el riego por goteo, lo que llevó al fracaso a esta empresa en su intento de consolidarse en el negocio del tomate industrial fue su mal manejo agronómico al intentar sembrar en forma constante el cultivo en una misma zona a lo largo de un año. Esta decisión trajo como consecuencia el incremento del problema fitosanitario, volviéndose incontrolable y muy costoso, con el resultado final del fracaso financiero.(7)

En el caso del pimiento páprika, que es una especie de la misma familia del tomate, también surgieron problemas fitosanitarios similares a los del tomate; sin embargo, en su incremento de sembríos por la década de 1990 pudieron ser exitosos porque no cometieron el error de sembrar ese cultivo en forma continua en un mismo lugar. Esto hizo que los problemas fitosanitarios resultaran manejables.

\section{El exitoso caso del Proyecto ASKA}

\section{Resumen del Proyecto ASKA}

Entre los años 2000 y 2002, los sectores industrial y académico llevaron a cabo el Proyecto de Restauración de la Industria Tomatera (Proyecto ASKA), en el que participaron empresas privadas japonesas, empresas peruanas y la Universidad Agraria Nacional La Molina del Perú, a fin de evaluar la factibilidad de volver a sembrar tomate industrial a campo abierto en escala comercial en la costa peruana.

Este proyecto se inició en marzo del 2000 en la zona de Trujillo, en un campo de cultivo alquilado de 10 ha. El objetivo fue evaluar la adaptabilidad de cultivares de tomate industrial y las tecnologías del manejo agronómico, así como la productividad y costos de producción empleando riego por goteo. La zona de Trujillo pertenece a un área especial de desarrollo agrícola, en el marco de un proyecto nacional 
de irrigación llevado a cabo por el gobierno (Proyecto Chavimochic para el desarrollo agrícola).

\section{Ensayo del cultivo del tomate para consumo} fresco a campo abierto a escala comercial

Se llevó de la siguiente manera:

a. Empleando riego por goteo entre diciembre del 2000 y marzo del 2001 (verano), se evaluaron 24 cultivares principales y 27 cultivares de menor importancia. Se obtuvieron en promedio $90 \mathrm{t} / \mathrm{ha}$.

b. Empleando riego por goteo entre los meses de julio y noviembre del 2001 (inviernoprimavera), se evaluaron 14 cultivares principales y 44 cultivares de menor importancia. Se logró producir en promedio 70 t/ha. c. Empleando riego por gravedad entre los meses de octubre del 2001 y febrero del 2002 (primavera-verano) se evaluaron 14 cultivares principales y 44 cultivares de menor importancia. Se logró producir en promedio $80 \mathrm{t} / \mathrm{ha}$.

\section{Resultados de las pruebas a campo abierto}

Los resultados de estas pruebas muestran la eficacia del riego por goteo en la consecución de altos rendimientos en siembras de tomate a campo abierto en la época de verano. Por otra parte, se evidenció que en la época de invierno, aun con goteo, el potencial de rendimiento se ve afectado. Esto implica retos aún por resolver en sembríos continuos de tomate en una misma zona desde el punto de vista técnico y económico (costos de producción). Los resultados de las diferentes pruebas se resumen en la tabla 1.

Tabla 1. Resultados de los ensayos de prueba de siembra de tomate industrial en el norte del Perú

\begin{tabular}{|c|c|c|c|}
\hline & $\begin{array}{l}\text { IRRIGACIÓN CON GOTEO. } \\
\text { CULTIVO DE VERANO }\end{array}$ & $\begin{array}{l}\text { IRRIGACIÓN CON } \\
\text { GOTEO. CULTIVO DE } \\
\text { INVIERNO }\end{array}$ & $\begin{array}{l}\text { RIEGO POR SURCO. } \\
\text { CULTIVO DE } \\
\text { PRIMAVERA }\end{array}$ \\
\hline $\begin{array}{l}\text { Condición de } \\
\text { clima }\end{array}$ & Nivel normal anual. & $\begin{array}{l}\text { Baja temperatura en } \\
\text { comparación con los años } \\
\text { normales anteriores. }\end{array}$ & $\begin{array}{l}\text { Baja temperatura en } \\
\text { comparación con los años } \\
\text { normales anteriores. }\end{array}$ \\
\hline $\begin{array}{l}\text { Control del } \\
\text { cultivo }\end{array}$ & $\begin{array}{l}\text { Se comprueban la alta } \\
\text { productividad y el control de } \\
\text { plagas. }\end{array}$ & $\begin{array}{l}\text { Se extiende la plaga debido al } \\
\text { fuerte viento. }\end{array}$ & $\begin{array}{l}\text { Se comprueban la alta } \\
\text { productividad y el control de } \\
\text { plagas. }\end{array}$ \\
\hline Variedad & Se seleccionan 12 variedades. & Se seleccionan 12 variedades. & Se seleccionan 12 variedades. \\
\hline $\begin{array}{l}\text { Cantidad de } \\
\text { cosecha }\end{array}$ & 90 a 100 t/ha. & $\begin{array}{l}60 \text { a } 70 \text { t/ha } \\
\text { (moho causado por } \\
\text { baja temperatura). }\end{array}$ & 75 a 85 t/ha. \\
\hline Calidad & $\begin{array}{l}\text { Bajo licopeno y contenido de } \\
\text { brix* }\end{array}$ & $\begin{array}{l}\text { Alto contenido de brix (baja } \\
\text { temperatura) y adecuado } \\
\text { contenido de licopeno. }\end{array}$ & $\begin{array}{l}\text { Bajo contenido de brix (por } \\
\text { lluvia) y adecuado contenido } \\
\text { de licopeno. }\end{array}$ \\
\hline $\begin{array}{l}\text { Costo de } \\
\text { producción }\end{array}$ & US \$ $3200 /$ ha & US \$ $3000 / h a$ & US \$ $3100 /$ ha \\
\hline $\begin{array}{l}\text { Competitividad } \\
\text { del costo }\end{array}$ & US $\$ 0,034 / \mathrm{kg}$ & US \$ 0,046/kg & US $\$ 0,039 / \mathrm{kg}$ \\
\hline
\end{tabular}

Fuente. Elaboración de los propios autores, con base en los datos de prueba.

* Unidad del grado de azúcar en la solución. 
Tal como se muestra en la tabla 2, comparando el Proyecto ASKA y las operaciones hechas por TOMANOR, ambas en la zona de Trujillo, se llega a la conclusión de que para ser exitosos en el cultivo de tomate industrial en la zona costera peruana se debe tener en cuenta que:

a. El sistema de riego por goteo es sumamente efectivo.

b. También resulta efectivo el Manejo Integrado de Plagas (MIP), por la reducción del uso de pesticidas que se logra en el control de plagas y enfermedades.

c. El uso de guano, sea de vacuno o de aves, es sumamente positivo, sobre todo por las características de la costa peruana debido a la mejora de las propiedades de los arenales. d. Comparando los costos por kilogramo producido, el proyecto ASKA es el más competitivo.

\section{El exitoso caso de la empresa ICATOM}

\section{Resumen operativo de la empresa ICATOM}

Entre los años 1995 y 2002, ICATOM hizo frente a la plaga de mosca blanca en sus campos de cultivo. ICATOM tomó la decisión de evaluar su proceso productivo de cultivos sucesivos observando, además, los resultados del Proyecto ASKA. Decide cultivar el tomate industrial solo una vez al año, lo que trae un incremento en sus rendimientos de $60 \mathrm{t} / \mathrm{ha}$ a 90-100 t/ha.

Tabla 2. Comparación entre el exitoso caso del Proyecto ASKA y casos de fracasos en el Perú

\begin{tabular}{|c|c|c|c|}
\hline & TOMANOR & AGROGUAYABITO & PROYECTO ASKA \\
\hline Irrigación & Irrigaciówn en gran escala. & Irrigación a goteo. & Irrigación a goteo. \\
\hline $\begin{array}{l}\text { Costo de } \\
\text { insecticida }\end{array}$ & US \$ $2500 /$ ha & US \$ $2000 /$ ha & US \$ $1200 /$ ha \\
\hline $\begin{array}{l}\text { Fertilizante } \\
\text { orgánico }\end{array}$ & Excremento de pollo. & Excremento de pollo. & $\begin{array}{l}\text { Excremento de res y guano } \\
\text { (excremento de ave } \\
\text { marina). }\end{array}$ \\
\hline $\begin{array}{l}\text { Frecuencia del } \\
\text { cultivo al año }\end{array}$ & Solamente en verano. & $\begin{array}{l}\text { Cultivo sucesivo anual. } \\
\text { Hay problema por el cultivo } \\
\text { continuo. }\end{array}$ & $\begin{array}{l}\text { Cultivo sucesivo anual. } \\
\text { No hay problema por el } \\
\text { cultivo continuo. }\end{array}$ \\
\hline Escala & 1000 ha. & 500 ha. & 10 ha. \\
\hline Lugar & Zona norte del Perú. & Zona central del Perú. & Zona norte del Perú. \\
\hline $\begin{array}{l}\text { Medidas contra } \\
\text { las plagas }\end{array}$ & $\begin{array}{l}\text { Uso intensivo de } \\
\text { insecticidas. }\end{array}$ & $\begin{array}{l}\text { Uso intensivo de } \\
\text { insecticidas. }\end{array}$ & $\begin{array}{l}\text { Aplicación del Manejo } \\
\text { Integrado de Plagas (MIP). }\end{array}$ \\
\hline $\begin{array}{l}\text { Cantidad de } \\
\text { cosecha }\end{array}$ & 30 a 50 ton/ha. & 50 a 70 ton/ha. & 90 a 100 ton/ha. \\
\hline Costo del cultivo & $\begin{array}{l}\text { US } \$ 4500 / \text { ha (costo } \\
\text { mínimo). }\end{array}$ & $\begin{array}{l}\text { US } \$ 4000 / \text { ha (costo } \\
\text { mínimo). }\end{array}$ & $\begin{array}{l}\text { US } \$ 3200 / \text { ha (costo } \\
\text { mínimo). }\end{array}$ \\
\hline $\begin{array}{l}\text { Competitividad } \\
\text { del costo }\end{array}$ & US $\$ 0,113 / \mathrm{kg}$ & US $\$ 0,067 / \mathrm{kg}$ & US $\$ 0,034 / \mathrm{kg}$ \\
\hline
\end{tabular}

Fuente. Elaboración de los propios autores, con base en los datos de prueba. 
Este es un caso donde una experiencia exitosa, como la del Proyecto ASKA, es tomada en cuenta para traer mejoras en el proceso de producción de tomate industrial a campo abierto.

\section{Comparación entre las operaciones de ICATOM y el proyecto ASKA}

\section{Comparando el Proyecto ASKA y las} operaciones de ICATOM, se pudieron identificar los siguientes puntos comunes:

a. Para el cultivo de tomate industrial a campo abierto, el empleo del riego por goteo es muy positivo. La principal fuente de agua es la subterránea, pero también está disponible la fluvial, producto de las lluvias de verano y de los glaciales de los Andes, en la época que no llueve.

b. Es necesario el empleo del MIP, ya que trae reducción en el empleo de agroquímicos. c. El empleo de guano (de vacuno o de aves) mezclado con los fertilizantes químicos es viable y positivo para la mejora de los rendimientos, sobre todo en las condiciones de la costa peruana.

\section{CONCLUSIONES}

En resumen, se puede afirmar lo siguiente:

1. Analizando el caso del pimiento páprika, se concluyó que el uso del riego por goteo es un factor preponderante para buenos rendimientos bajo las condiciones de la costa peruana en escala comercial (se debe recordar que el Perú está situado entre los 0 y 30 grados de latitud sur). Además, se concluye que otro aspecto de importancia para el éxito del cultivo de tomate industrial es el manejo

\section{Tabla 3. Comparación entre el Proyecto ASKA y las operaciones de ICATOM}

\begin{tabular}{|c|c|c|}
\hline & ASKA (Zona norte del Perú) & ICATOM (Zona sur del Perú) \\
\hline Irrigación & Irrigación por goteo. & $\begin{array}{l}\text { Irrigación por goteo (principal). } \\
\text { Irrigación por surco (secundario). }\end{array}$ \\
\hline $\begin{array}{l}\text { Frecuencia del cultivo } \\
\text { anual }\end{array}$ & Cultivo sucesivo anual. & $\begin{array}{l}\text { Una vez al año (cultivo en invierno- } \\
\text { primavera). }\end{array}$ \\
\hline Manejo de plagas & $\begin{array}{l}\text { Reducción del uso de insecticidas. } \\
\text { Aplicación del MIP. }\end{array}$ & $\begin{array}{l}\text { Reducción del uso de insecticidas, } \\
\text { combinado con el cultivo orgánico. }\end{array}$ \\
\hline Escala & 10 ha. & 100 ha. \\
\hline Fuente de agua & $\begin{array}{l}\text { Agua de río (lluvia o nieve derretida de } \\
\text { los Andes). }\end{array}$ & Agua subterránea \\
\hline Fertilizante orgánico & Excremento de res y guano. & Excremento de pollo y guano. \\
\hline $\begin{array}{l}\text { Costo del uso de } \\
\text { insecticidas }\end{array}$ & US $\$ 1$ 200/ha. & US $\$ 900 /$ ha. \\
\hline Cantidad de cosecha & 90 a $100 \mathrm{t} / \mathrm{ha}$. & $\begin{array}{l}100 \text { a } 120 \text { t } / \text { ha (solo con riego por } \\
\text { goteo). }\end{array}$ \\
\hline Costo de cultivo & US \$ 3 200/ha (costo mínimo). & $\begin{array}{l}\text { US } \$ 2900 / \text { ha (solo con riego por } \\
\text { goteo). }\end{array}$ \\
\hline Competitividad del costo & US $\$ 0,034 / \mathrm{kg}$ & US $\$ 0,026 / \mathrm{kg}$ \\
\hline
\end{tabular}

Fuente. Elaboración de los propios autores, con base en los datos de prueba. 
de plagas buscando la reducción del empleo de agroquímicos en el cultivo.

2. Para el caso del cultivo del tomate industrial, se concluye que el riego por goteo también es pieza clave en el éxito del cultivo a escala comercial. Son ventajas adicionales las condiciones de clima de la costa peruana, el mismo que es moderado a lo largo del año, así como la disponibilidad de agua subterránea y de origen fluvial procedente de los Andes peruanos.

3. Comparando los casos de fracaso y de éxito en el cultivo del tomate industrial en la costa peruana, podemos señalar las siguientes ventajas:

a. El uso de guano (de vacuno o de ave) contribuye a la mejora de los rendimientos.

b. Existen muchas áreas aún no explotadas agrícolamente con suelos no contaminados y fértiles que con una adecuada rotación de cultivos y el manejo integrado de plagas se pueden incorporar a la agricultura. Además, el suelo es poco contaminado y muy fértil.

c. Debe realizarse un solo cultivo al año para evitar problemas de incremento de plagas y enfermedades.

d. Es factible el cultivo a gran escala por la facilidad de conseguir mano de obra calificada.

Aunque no se menciona en esta investigación, existe el potencial de incrementar las áreas de siembra de tomate industrial a campo abierto en la zona baja tropical situada entre los 0 y 30 grados de latitud norte y sur, mediante el uso simultáneo de una tecnología de ahorro de agua desarrollada por la Universidad de Agricultura de Tokio, en Djibouti (denominada Water Harvesting Stone Multi Engineering), lugar situado en la zona baja tropical, ubicada entre los 0 y 30 grados de latitud norte.

\section{Palabras de agradecimiento}

Quisiéramos expresar nuestro agradecimiento a la empresa Kagome Co. Ltd. y a la empresa Alicorp por la generosidad de habernos permitido el uso de los datos concernientes al cultivo del tomate a campo abierto en el Perú.

\section{REFERENCIAS BIBLIOGRÁFICAS}

1. FAO. The State of Food and Agriculture 20102011. Roma, 2011.

2. R. Angelini. Pomodoro. ART Servizi Editoriali S.p.A., Italia, 2010.

3. Heuvelink, E. Tomatoes. CABI Publishing, Inglaterra, 2005.

4. Delgado de la Flor, F., R. Ugas and A. Casas. Datos básicos de cultivos hortalizas. Univ. Nac. Agraria La Molina, 2000.

5. Instituto Peruano del Espárrago y Hortalizas (IPEH). Manual del cultivo de páprika. 2006.

6. L. G. James. Principles of Farm Irrigation System design. John Wiley and Sons, E.U.A., 1988.

7. A. Casas. Jefe del Dpto. de Horticultura de la Univ. Nac. Agraria La Molina. Comunicación personal, 2010. 


\section{ANEXO 1}

\section{Fotografías}

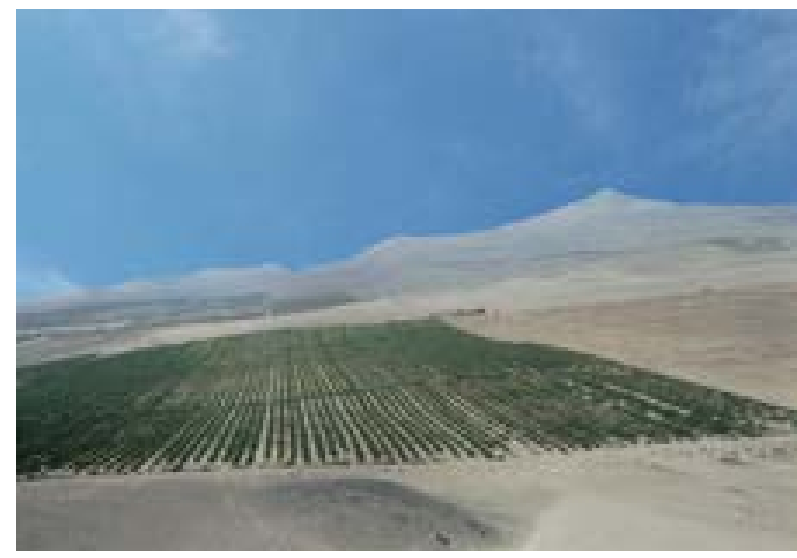

Figura 1. Desierto de la zona costera en el norte del Perú y dentro del paisaje un campo de cultivo de tomate.

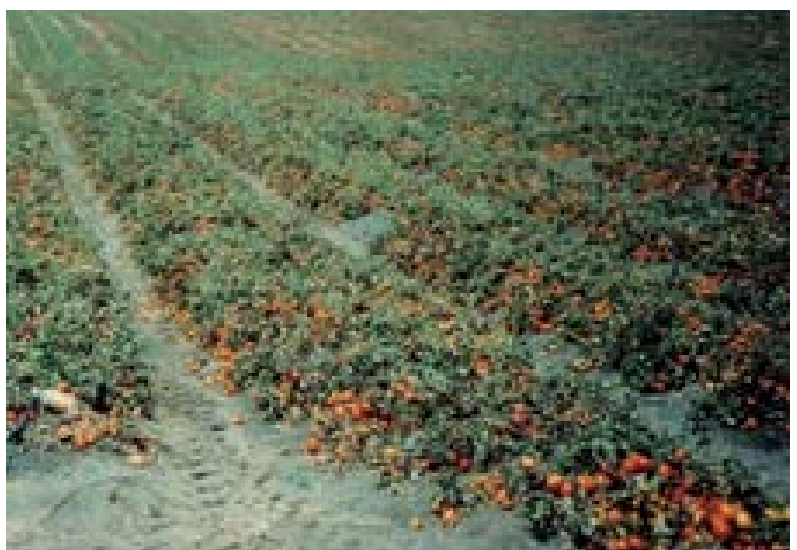

Figura 2. Campo de tomates en el desierto de la zona costera norte del Perú.
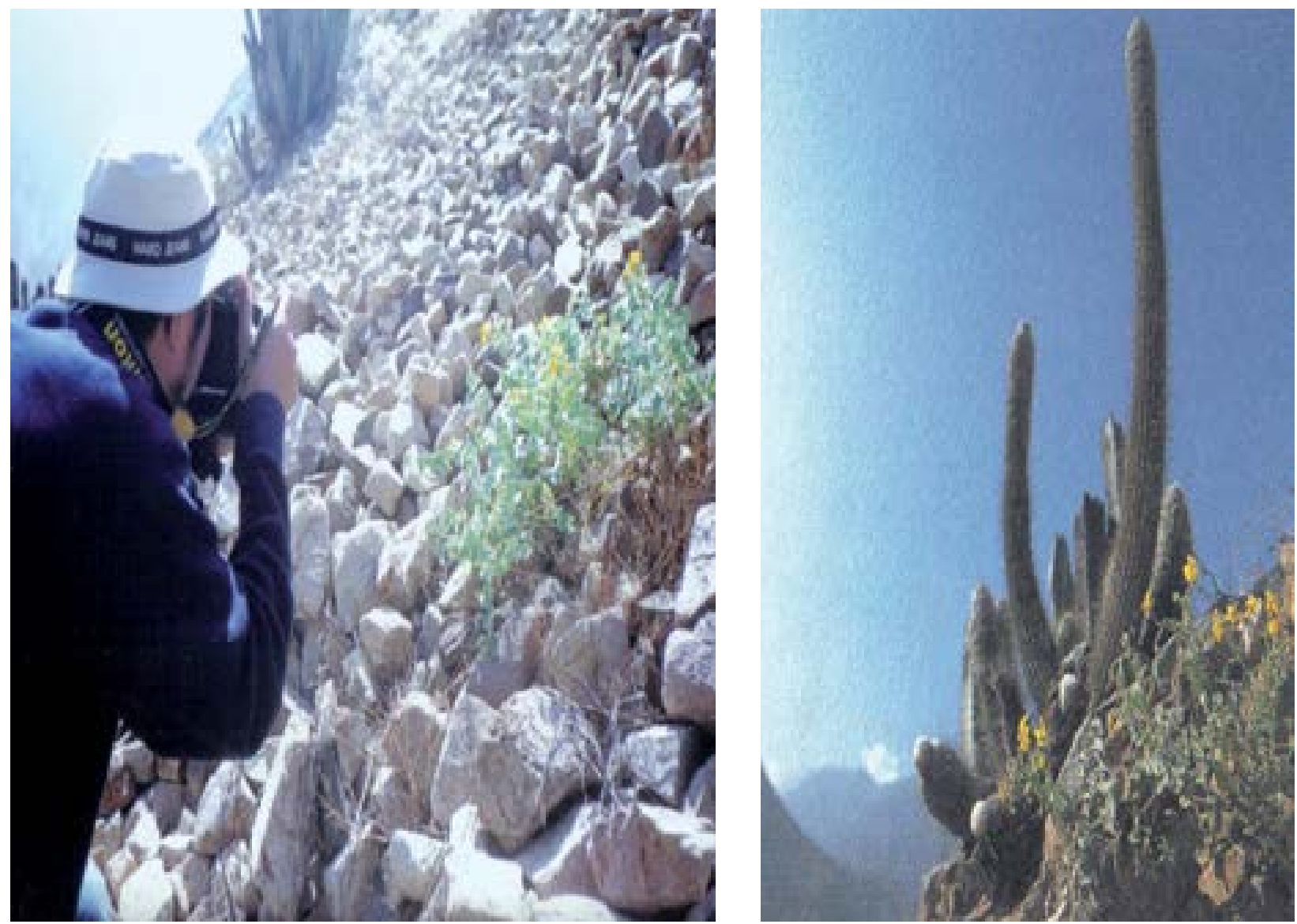

Figura 3. Tomate silvestre del Perú. 

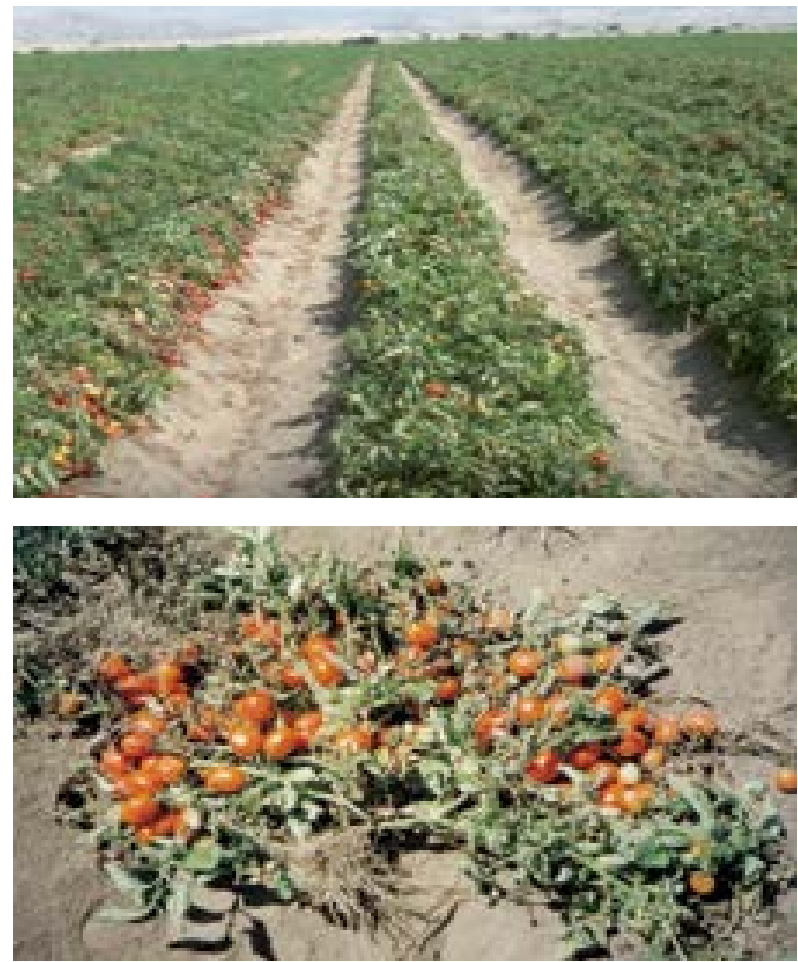

Figura 4. Imágenes del cultivo de tomate por

ICATOM en el desierto de la zona costera sur del Perú. (Fotografía tomada por el autor en el año 2005.)
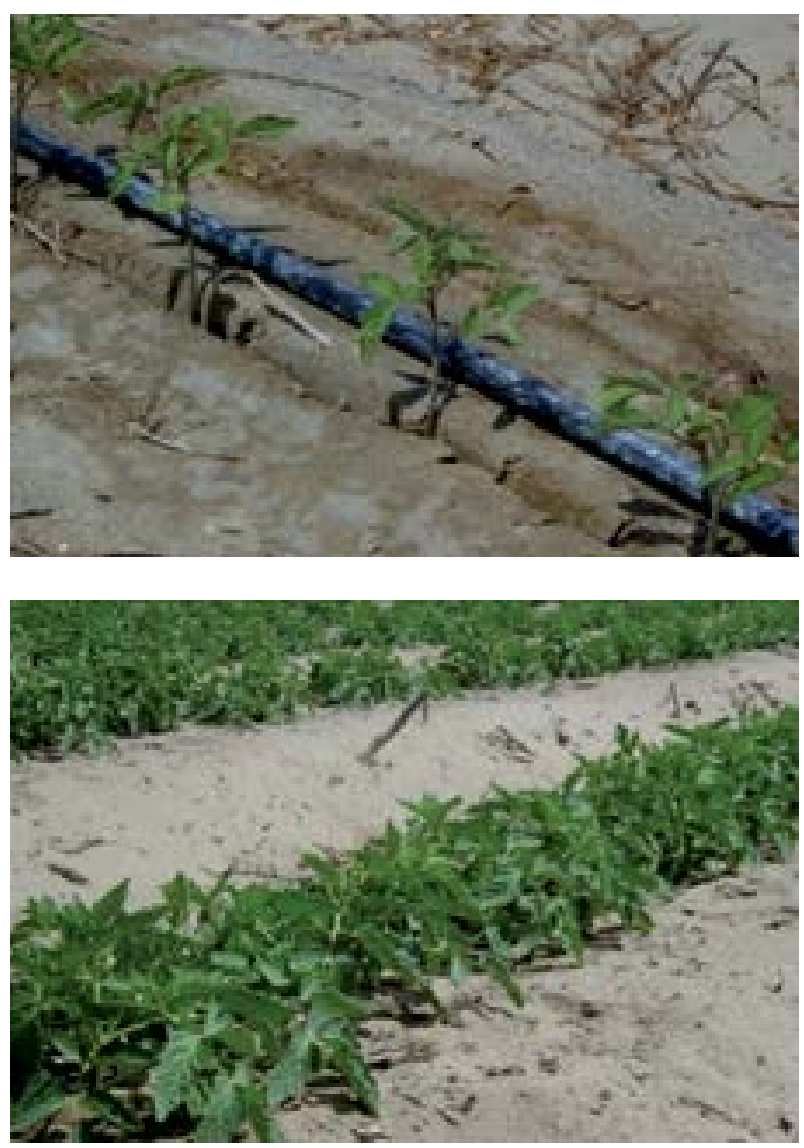

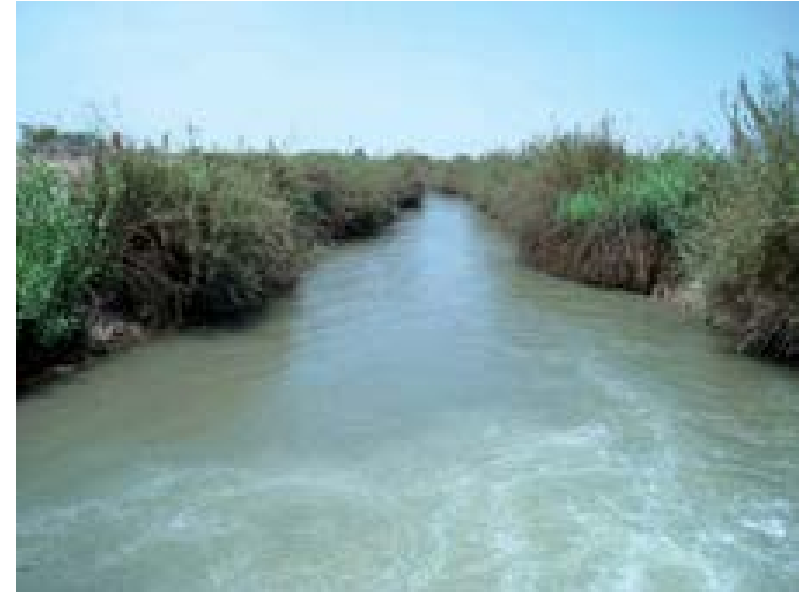

Figura 5. Canal de riego en el área de Ica (Perú), construido en la época incaica (hace 5 siglos) y que durante todo el año provee abundante agua proveniente de los Andes (lluvias y deshielo).
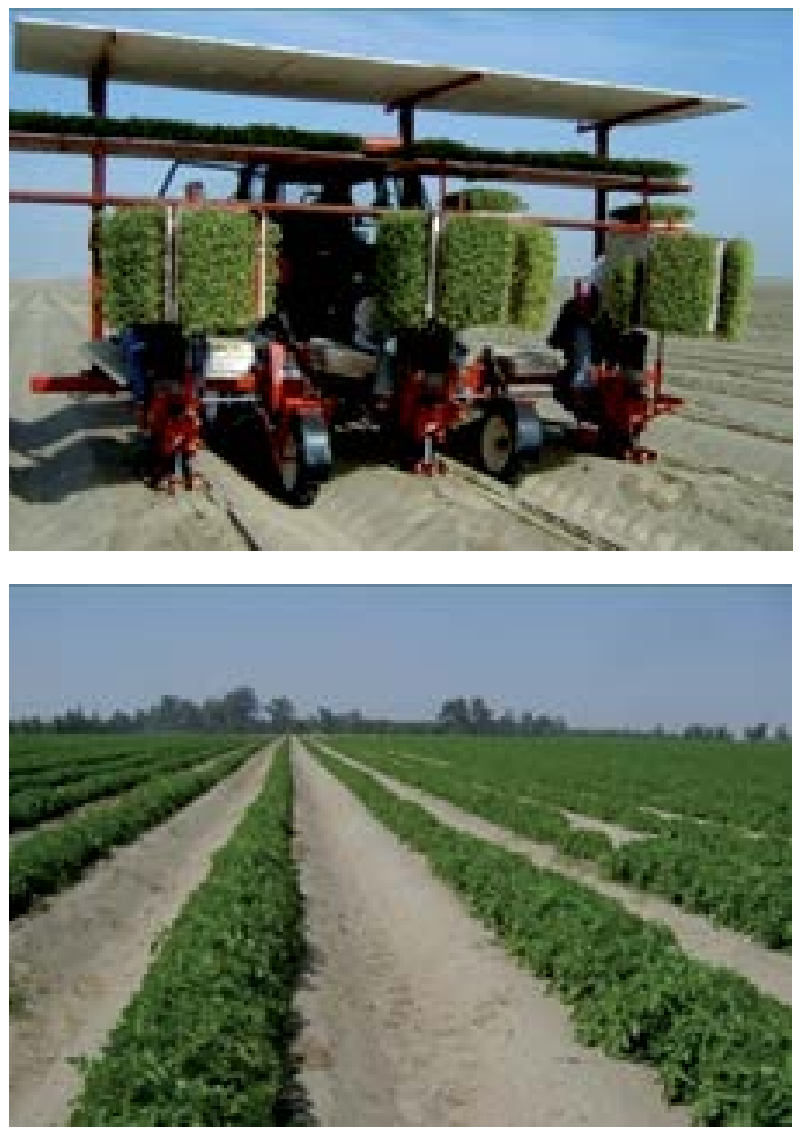

Figura 6. Cultivo del tomate a campo abierto empleando la agricultura con pocos insumos (uso del riego por goteo, manejo integrado de plagas y uso de materia orgánica). 